\title{
Demonstration of PECVD SiC thermal delay lines for optical coherence tomography in the visible
}

\author{
Grégory Pandraud $^{\mathrm{a}}$, Eduardo Margallo-Balbás ${ }^{\mathrm{b}}$ and Pasqualina M. Sarro ${ }^{\mathrm{a}}$ \\ ${ }^{a}$ Laboratory of Electronic Components, Materials and Technology, TU Delft, \\ Feldmannweg 17, 2628CT, Delft The Netherlands \\ ${ }^{b}$ Electronic Instrumentation Laboratory, TU Delft, Mekelweg 4, 2628CD, Delft, The \\ Netherlands
}

\begin{abstract}
Optical Coherence Tomography (OCT) has found applications in many fields of medicine and has a large potential for the optical biopsy of tumors. One of the technological challenges impairing faster adoption of OCT is the relative complexity of the optical instrumentation required, which translates into expensive and bulky setups. In this paper we report an implementation of Time-Domain Optical Coherence Tomography based on Plasma Enhanced Chemical Vapor Deposition (PECVD) Silicon Carbide (SiC). The devices, with a footprint of $0.3 \mathrm{~cm}^{2}$, are fabricated using rib waveguides defined in a $\mathrm{SiC}$ layer. While most of the components needed are known when using this material [1], a fast delay line with sufficient scanning range is a specific requirement of Time Domain (TD)-OCT. In the system reported here this is obtained making use of the thermo-optical effect. Though the current implementation still requires external sources and detectors to be coupled to the planar waveguide circuit, future work will include three-dimensional integration of these components onto the substrate to achieve a fully autonomous and compact OCT chip. With the potential to include the read-out and driving electronics on the same die, the reported approach can yield extremely compact and low-cost TD-OCT systems in the visible, enabling a broad range of new applications, including OCT devices for harsh environment.
\end{abstract}

Keywords: Optical Coherence Tomography, Silicon Carbide, Thermo-Optical Effect, Planar Waveguide Circuits

\section{INTRODUCTION}

Optical Coherence Tomography (OCT) is an imaging modality able to probe tissue at high resolution using infrared light [2]. Although its penetration depth is limited, it has been successfully applied to fields ranging from ophthalmology [3] to cardiology [4]. Modern OCT instrumentation is normally based on fibre optics, which allows for robust, portable and relatively compact assemblies, suitable for general clinical use. Despite the advantages gained from optical fibre equipment relative to free-space optics, current OCT instrumentation is still too bulky and costly for many applications. In fact, it can be argued that these two factors are impairing faster adoption of the technology in many fields of medicine. In some cases where footprint and price are key requirements, this is particularly obvious. In the case of smart-pills, much benefit could be gained from an on-board OCT-based optical biopsy system. However, the limited available space completely forbids this idea using current technology. In many fields of medicine but especially for surgical guidance, the information gained from OCT can be very useful, but does not justify the high cost of the technology.

In order to tackle these two shortcomings of current OCT implementations, namely cost and size, we have designed an ultra-compact TD-OCT engine based on SiC planar waveguides suitable for batch manufacturing. Reports [5] on PECVD SiC optical waveguide has shown that acceptable losses with a good beam confinement in the guiding core layer are achievable in the visible. As Si cannot be used in the visible the use of $\mathrm{SiC}$ will pave the way to new range applications working in a region where light sources are much cheaper to produce. The mechanical strength of the material makes it also attractive for use at

Biophotonics: Photonic Solutions for Better Health Care II, edited by Jürgen Popp,

Wolfgang Drexler, Valery V. Tuchin, Dennis L. Matthews, Proc. of SPIE Vol. 7715, 771509

(C) 2010 SPIE · CCC code: 1605-7422/10/\$18 - doi: 10.1117/12.853686

Proc. of SPIE Vol. $7715771509-1$ 
high optical power densities like the one provided by Raman sources as well as in harsh environment. Further by working in the visible the resolution of OCT systems can be significantly improved [6]. Furthermore, the low deposition temperature allows the compatibility with standard CMOS process and therefore the subsequent integration of read out electronics.

\section{PECVD SiC WAVEGUIDES PROPERTIES}

In the following delay lines are fabricated using straight waveguides. The choice of $\mathrm{SiC}$ can be justified by looking at the $(\mathrm{n}, \mathrm{k})$ curves presented in Fig 1 and obtained by performing ellipsometry on a $200 \mathrm{~nm}$ thick PECVD SiC film deposited on a Si substrate. They show that the films are transparent in the visible $(\mathrm{k}=0$ from $600 \mathrm{~nm}$ ) with a refractive index higher than 2.4 in the same region of the spectrum. Further the thermal properties of $\mathrm{SiC}$ reported in Table 1 show that the thermo optical coefficient even if lower than the one of $\mathrm{Si}$ can allow the production of delays. By comparison the thermo optical coefficient of $\mathrm{SiO}_{2}$ is 4 times smaller.

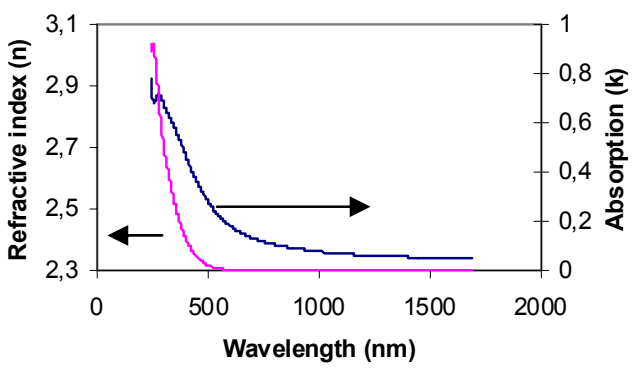

Fig. 1. Refractive index and absorption of PECVD SiC

\begin{tabular}{|l|l|}
\hline \multicolumn{1}{|c|}{ Property } & \multicolumn{1}{c|}{ Value } \\
\hline $\begin{array}{l}\text { Thermo optical } \\
\text { coefficient }\end{array}$ & $3.710^{-5} / \mathrm{K}$ \\
\hline Thermal diffusivity & $<10^{-2} \mathrm{~cm}^{2} \mathrm{~s}^{-1}$ \\
\hline Thermal conductivity & $130-160 \mathrm{Wm}^{-1} \mathrm{~K}^{-1}$ \\
\hline
\end{tabular}

Table 1. Thermal properties of $\mathrm{SiC}$ obtained by ellipsometry.

\section{1) Propagation losses}

One of the key requirements for the delay lines in order to be used efficiently in an OCT system is a low propagation loss of the waveguides. Fig. 2 presents the set up that was used to test the delay lines once fabricated. The light when it reaches the component will cross it once and after reflection on the end face of the device cross it a second time. In order to achieve a significant delay as explain later the component have a long section where heat is applied. To get a readable signal on the detectors it is therefore critical that the light does not attenuate too much in the chip. Further as one of the goal of the work is to achieve a fully integrated system it is also obvious that not only the long delay lines have to be low loss but the $2 \times 2$ coupler too.

To investigate those losses, we first deposited a $2 \mu \mathrm{m}$ thick $\mathrm{PECVD} \mathrm{SiO}_{2}$ layer on a standard $\mathrm{Si}$ wafer. The oxide serves to optically isolate the circuit from the substrate and reduce the loss due to substrate leakage. Then a $2 \mu \mathrm{m}$ thick PECVD SiC layer was deposited using silane $\left(\mathrm{SiH}_{4}\right)$ and methane $\left(\mathrm{CH}_{4}\right)$ in a Novellus Concept One system. The temperature of deposition was $400{ }^{\circ} \mathrm{C}$. Wafers were coated with photoresist and exposed using an ASML stepper. $3 \mu \mathrm{m}$ wide singlemode straight waveguides were etched down to $1.6 \mu \mathrm{m}$ in Trikon Omega inductively coupled plasma with: $30 \mathrm{sccm} \mathrm{Cl}_{2}$, and $15 \mathrm{sccm} \mathrm{HBr}$ under 9.5 Torr at $25^{\circ} \mathrm{C}$, and with an ICP RF power of $500 \mathrm{~W}$ and a RF platen power of $35 \mathrm{~W}$. This process allows a etching uniformity over a 4 inch wafer of $390 \pm 15 \mathrm{~nm}$ and a waveguide width uniformity of $3 \pm 0.5 \mu \mathrm{m}$. The estimated etch rate over the entire wafer is $380 \mathrm{~nm} / \mathrm{min}$. $\mathrm{SiC}$ is often seen as a pretty difficult material to etch and often RIE processes need to be tuned to get very smooth sidewalls compatible with photonics [7]. In the following no specific adjustment were made to the etching process and a SEM view of a fabricated waveguide is shown in Fig . 3 a). The waveguides are finally covered with a $2 \mu \mathrm{m}$ thick PECVD $\mathrm{SiO}_{2}$ layer. This step has two purposes. First of all this layer will prevent leakage to the electrodes deposited on the top 
of the waveguides to modulate the refractive index using heat and secondly it allows a reduction of the propagation losses that are proportional to the square of the waveguide contrast (cladding/core).

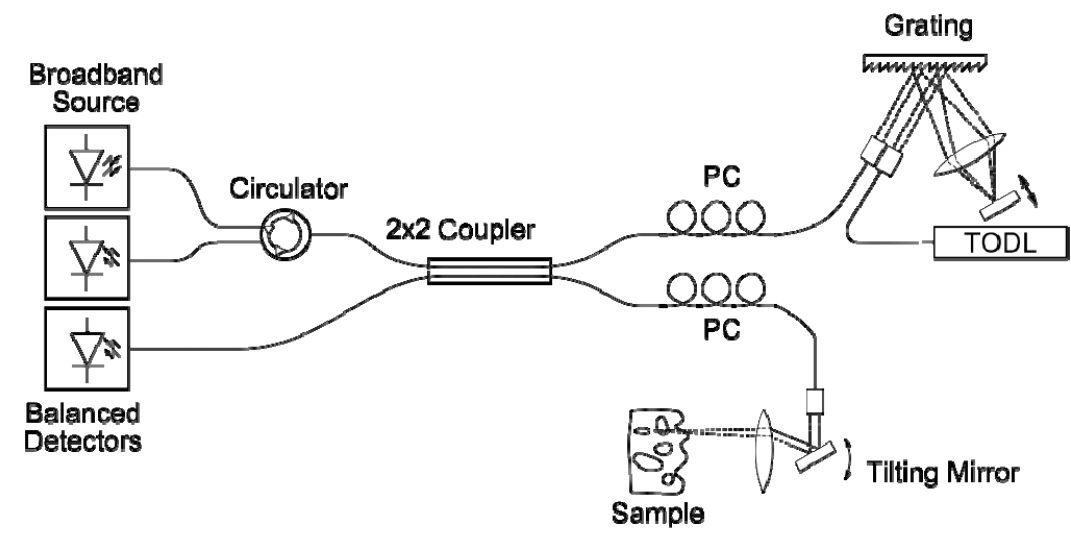

Fig. 2 Schematic of the OCT system, including a grating based chromatic dispersion compensation stage and balanced detection. For the characterisation of the devices, a fixed mirror in the sample arm was used.

The losses are estimated using the cut back method which consists of measuring the transmission of different waveguide lengths. When plotting the transmission versus the length of each tested segments, the loss per unit length can be found by linearly fitting the different point obtained. The losses of different PECVD SiC waveguide lengths $\mathrm{SiC}$ at wavelength of $1.3 \mu \mathrm{m}$ are depicted in Fig. $3 \mathrm{~b}$ ). The linear fit for both TE and TM polarizations gives a slightly negative slope that has no physical meaning. The propagation losses for this type of waveguide are too low to be measured accurately and are with all probability under $1 \mathrm{~dB} / \mathrm{cm}$. It is also interesting that polarization dependent losses are correspondingly very low.

The roughness of the $\mathrm{SiC}$ waveguides vertical sidewall [8] was found to be $5.7 \mathrm{~nm}$ (RMS) with a mean correlation length over the whole vertical surface averaged of $243 \mathrm{~nm}$. Being a PECVD layer, the surface roughness of the as-deposited $\mathrm{SiC}$ layer is comparable to that value confirming then that the etching process used to produce the waveguide is good enough.

When inserting these measured value into the Payne and Lacey s model [9] (conventional used to give an estimation of the scattering losses in optical waveguides), the expected simulated losses are about to be 1 $\mathrm{dB} / \mathrm{cm}$ at $1.3 \mathrm{um}$. This value is in good agreement with the measured one and if we look at the losses at other wavelength, for example in the visible, using the same model simulated losses are around $5 \mathrm{~dB} / \mathrm{cm}$. This value is in agreement with the losses reported in [5] and confirms that the PECVD SiC is a good candidate for low loss delays lines in the visible.

\section{2) Chromatic dispersion}

The wavelength dependency of the refractive index of $\mathrm{SiC}$ induces sufficient material chromatic dispersion to significantly impact the final resolution of the OCT system for a standard super-luminescent diode source. If we assume that there is no dispersion in the sample arm the group delay dispersion (i.e. the second order term in the wavelength dependency) corresponds to a broadening of the temporal Point Spread Function (PSF) equal to:

$$
\tau_{c r i t}=\sqrt{G D D}
$$

The broadening of the Gaussian envelope of the interferometric signal is in turn given by

$$
2 c \sigma_{\tau}=2 c \sigma_{\tau} \sqrt{1+\left(\frac{\tau_{c r i t}}{\sigma_{\tau}}\right)^{4}}
$$


where $\sigma_{\tau}$ represents the Gaussian envelope for a non-dispersive system caused by the limited bandwidth of the source and $\sigma_{\tau}$ is the new effective envelope width parameter. The value of $\sigma_{\tau}$ can be computed from the specifications of the limited coherence source as

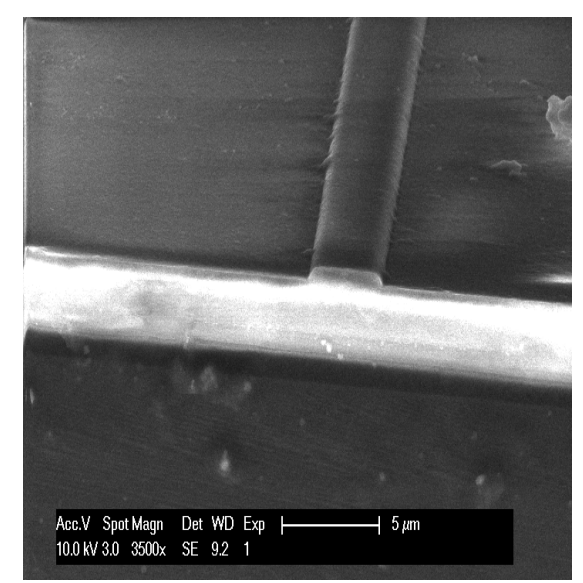

a)

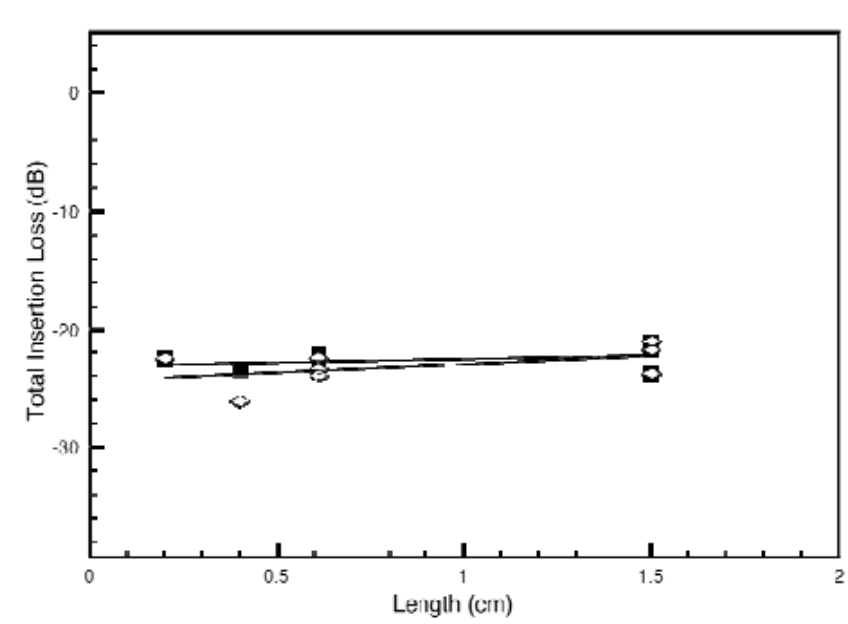

b)

Fig. 3. a) SEM view of the fabricated waveguides ; b) the corresponding transmission losses (squares TE circles TM)

$$
\sigma_{\tau}=\frac{\lambda^{2}}{2 \pi c \Delta \lambda}
$$

The source used in the set up presented in Fig. 2 has a bandwidth of $50 \mathrm{~nm}$ and a center wavelength of 1300 $\mathrm{nm}$ so $\sigma_{\tau}=17.9 \mathrm{fs}$ corresponding to a spatial envelope parameter of $10,7 \mu \mathrm{m}$. The $\mathrm{SiC}$ waveguides fabricated here considering only the second order term in the refractive index function $\tau_{\text {crit }}=92 \mathrm{fs}$ expanding the PSF by $325 \mu \mathrm{m}$ and thus deteriorating the system resolution.

A double-pass grating system based chromatic dispersion compensation scheme [10] was then used to correct the material dispersion (Fig .2) By changing a geometrical parameter in the setup, any dispersion value and sign can be generated. A group delay dispersion of opposite sign to the one stemming from the thermo-optic delay line can be introduced. In the future we hope to be able to compensate these kind of dispersion on chip.

It is worth noticing here that when moving towards the visible $\sigma_{\tau}$ becomes even smaller making then the cancelling of the dispersion a key issue.

\section{3) Birefringence}

The TM and TE modes in the waveguide have different group velocities and as small this difference is it is sufficient to alternate from constructive to destructive interference between both modes and cause distortions to the shape of the Gaussian envelope shape. Therefore birefringence management is as crucial as the chromatic dispersion compensation.

The waveguides fabricated for the losses measurements are depicted schematically in Fig. 4. Here the rib height $\mathrm{H}=2 \mu \mathrm{m}$, the width $\mathrm{W}=3 \mu \mathrm{m}$ and the rib edge depth $\mathrm{H}-\mathrm{h}=0.4 \mu \mathrm{m}$.

As reported elsewhere [11], the birefringence of such waveguides $\left(\Delta \mathrm{n}_{\text {eff }}=\mathrm{n}_{\text {eff }}^{\mathrm{TM}}-\mathrm{n}_{\text {eff }}^{\mathrm{TE}}\right)$ is a combination of geometrical and cladding stress induced birefringence. For the waveguide dimensions given above and a refractive index of $\mathrm{SiC}$ of 2.35 (from Fig. 1), the waveguides have a large geometrical birefringence of 
$\Delta \mathrm{n}_{\mathrm{eff}}=3 \times 10^{-3}$. Now one of the benefit of working with layers deposited at low temperature is that annealing can be applied with success. As shown in Fig. 5, after depositing the $2 \mu \mathrm{m} \mathrm{SiC} \mathrm{films} \mathrm{on} \mathrm{the} \mathrm{SiO}_{2}$ lower cladding we performed a strain measurement and looked at how it can be controlled by annealing.

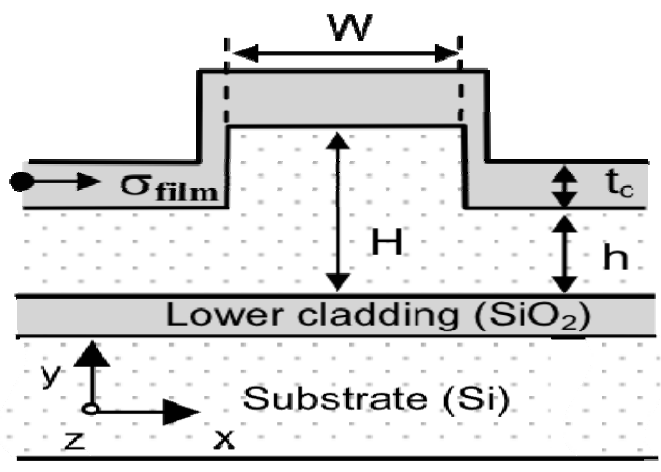

Fig. 4 Schematic cross section of a PECVD SiC rib waveguide with the key parameters required in the birefringence process

As reported elsewhere [11], the birefringence of such waveguides $\left(\Delta \mathrm{n}_{\text {eff }}=\mathrm{n}_{\text {eff }}^{\mathrm{TM}}-\mathrm{n}_{\text {eff }}^{\mathrm{TE}}\right)$ is a combination of geometrical and cladding stress induced birefringence. For the waveguide dimensions given above and a refractive index of $\mathrm{SiC}$ of 2.35 (from Fig. 1), the waveguides have a large geometrical birefringence of $\Delta \mathrm{n}_{\text {eff }}=3 \times 10^{-3}$. Now one of the benefit of working with layers deposited at low temperature is that annealing can be applied with success. As shown in Fig. 5, after depositing the $2 \mu \mathrm{m} \mathrm{SiC} \mathrm{films} \mathrm{on} \mathrm{the} \mathrm{SiO}_{2}$ lower cladding we performed a strain measurement and looked at how it can be controlled by annealing.

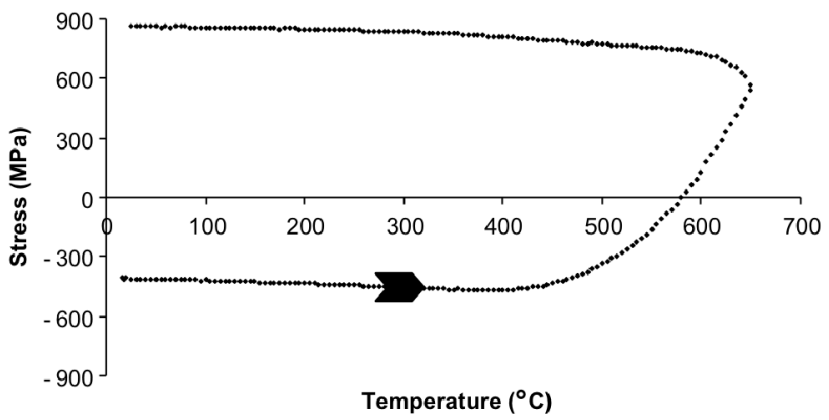

Fig .5 Stress in a PECVD SiC film on oxide during annealing

Fig. 5 shows that there is a transition of the strain from one state to another state, from example from compressive to tensile. Therefore it s possible to achieve stress free waveguides at first by annealing the waveguides between 500 and $600{ }^{\circ} \mathrm{C}$ and control the residual geometrical birefringence by adjusting the upper cladding layer thickness $t_{c}$. The stress induced birefringence scales linearly with the stress level $\sigma_{\text {film }}$ in the cladding and increases with the cladding thickness $t_{c}$. The calculated birefringence versus the cladding thickness gave for a typical $\mathrm{SiO}_{2}$ stress level of $\sigma_{\text {film }}=-200 \mathrm{MPa}$ (compressive) a zero birefringence for a $\mathrm{t}_{\mathrm{c}}$ of $1.5 \mu \mathrm{m}$. The later while fabricating the delay lines this thickness was taken to isolate the $\mathrm{SiC}$ core from the metal electrodes. 


\section{PECVD SiC DELAY LINES}

Fig. 6 shows a fabricated fast scanning delay line. It is manufactured using the rib waveguides defined in $\mathrm{SiC}$ using the procedure detailed in the previous section. The final chip has a footprint of $0.3 \mathrm{~cm}^{2}$.

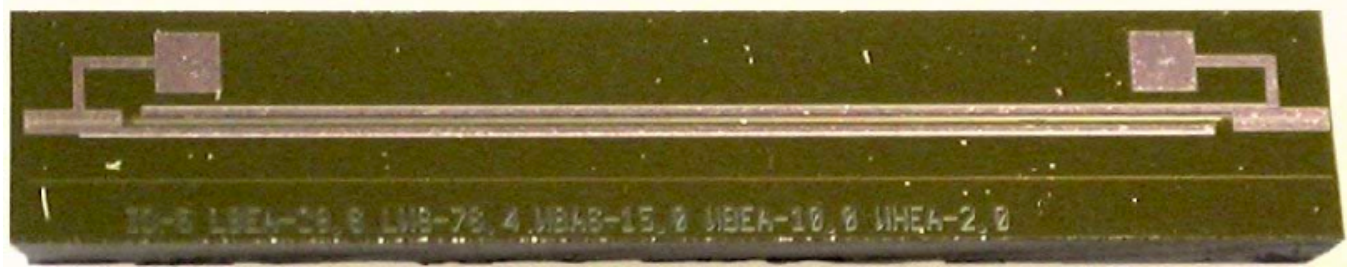

Fig. 6 Close up view of the fabricated delay lines. The chips are $27 \mathrm{~mm}$ long

This component makes use of the thermo-optical effect of $\mathrm{SiC}$ [12]. In some devices, the end of the reference arm was terminated using a metalized fiber to enhance reflection at the waveguide end. In other cases, the end was left open so that an arbitrary fixed delay could be added. In this implementation, sources and detectors have to be connected to obtain a full OCT engine. This is achieved by means of an intermediate butt-coupled optical fibre. The sample arm (Fig. 2) is also accessible for connection to any desired focusing system. The fast-scanning delay line is based on a freestanding $\mathrm{SiC}$ waveguide that can be thermally cycled at high-speed. Fig. 7 shows a cross section of the chip active area of this device.

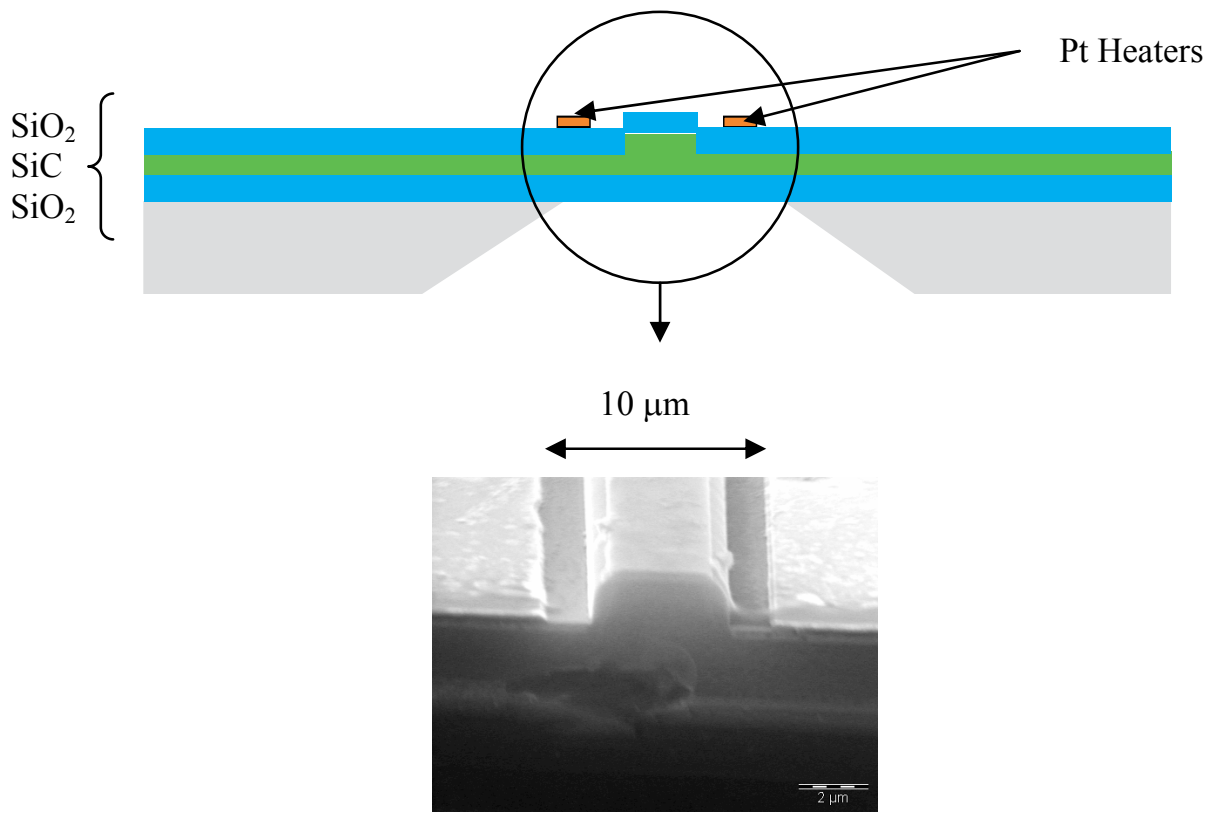

Fig. 7 Schematic of the cross section of the fabricated delay lines with a SEM close up view of the waveguides surrounded by heaters.

Because of the thermo-optical effect in $\mathrm{SiC}$, changes in core temperature are immediately translated into changes in refractive index and therefore a continuously varying path length can be obtained. A threemasks process has been developed for manufacturing the devices. 
On the $\mathrm{SiC}$ waveguides with a cladding of $1.5 \mu \mathrm{m}$ PECVD oxide, two evaporated Pt heaters are defined by means of a lift off process (Fig. 8). A negative resist is first spun on the wafer (Fig.8 b)) and patterned. Then Pt is evaporated and the wafers dropped in acetone to leave only the heaters on the wafer. These heaters provide thermal actuation to the structure but are also used as sensors to monitor the temperature of the wave guiding region by measuring their instantaneous electrical resistance during operation. The heater is structured in sections connected in parallel. This makes the parasitic resistance of the conduction lines from the connection point to the actual heater section quite critical. It is very important at his stage that the evaporation of the first metal is done at an angle $\left(>45^{\circ}\right)$ to ensure that the metal deposits nicely over the rib and connect every sections. However in order to reduce the effect of the parasitic resistance, a second metal layer has been added (Fig. 8 d)). For this layer, gold is used instead of platinum because of its lower resistance. Its thermal and reliability properties are not so crucial, because it is not deposited close to the hot line in the membrane. The membranes are created by means of $\mathrm{KOH}$ etching from the back side of the wafer using a nitride mask and protecting the front side (to prevent any etching of the heaters in $\mathrm{KOH}$ ). The etching is stopped when the oxide layer is reached.

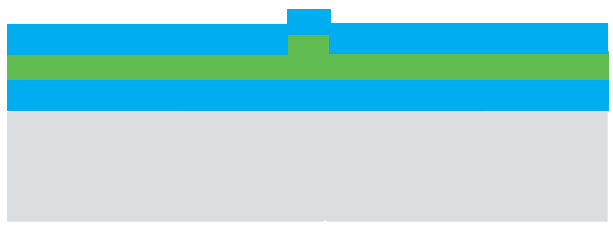

a)

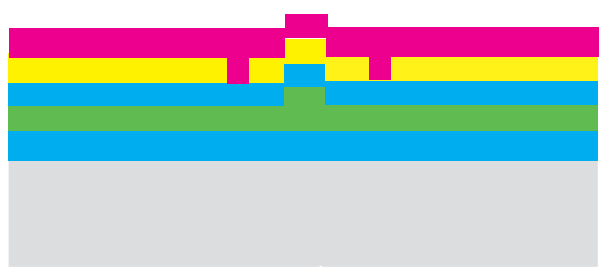

c)

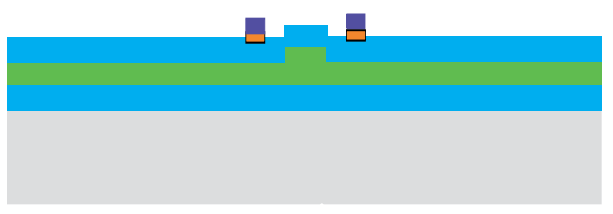

e)

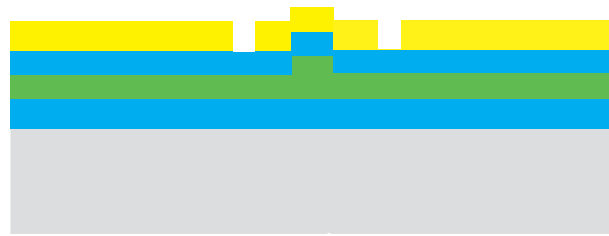

b)

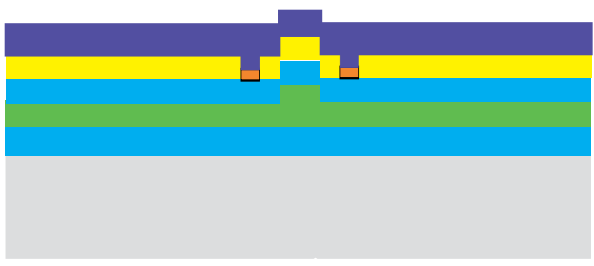

d)

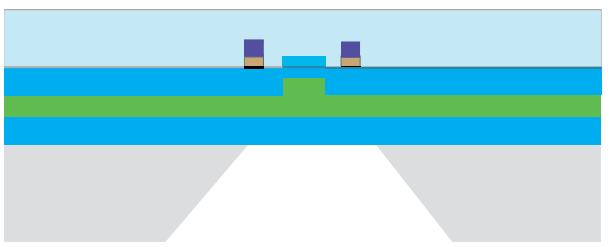

f)

Fig. 8. Schematic process flow to produce the delay line from a birefringence free PECVD SiC waveguide

The thermal properties of the membranes defines the frequency behaviour of the final devices, and it is therefore of utmost importance. We characterized this behaviour applying a step response and monitoring the change of heater resistance over time, which is known to be approximately proportional to the temperature deviation. Fig. 9 contains a schematic depiction of the measurement setup. The excitation signal was a pulse train with a frequency of $10 \mathrm{~Hz}$ and a peak power of $250 \mathrm{~mW}$. The upper resistor represents the heater, with a value that is a function of temperature. This dependency is expressed under a linearity assumption in the value of the component, where $\alpha$ is the temperature coefficient of the metal. The lower element is a sense resistor to measure the current. By measuring the voltage over the sense resistor we get a measure of the intensity through the heater, while we monitor the applied voltage in $V_{1}$. 
Operating on the electrical network in Fig. 9, we come to the expression of temperature as a function of the two measurable voltages in the schematic that is contained in (4).

$T=\frac{1}{\alpha}\left\lfloor\left(\frac{V_{1}}{V_{2}}-1\right) \frac{1}{R}-1\right\rfloor$

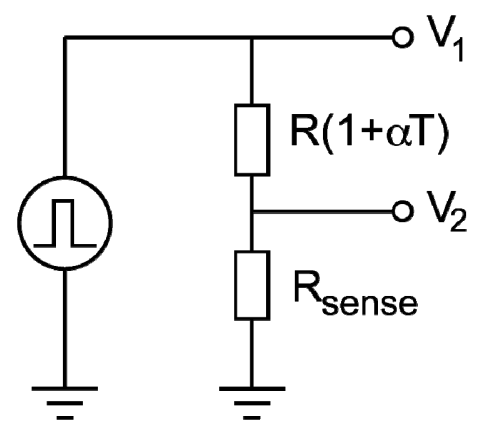

Fig. 9. Schematic representation of the measurement configuration for characterizing the electro-thermal behavior of the delay lines.

\section{RESULTS AND DISCUSSION}

Fig. 10 shows the thermal behaviour obtained using the setup described above for two membrane sizes. The continuous lines are the measured parameters, while the broken lines correspond to the best-fitting parameters of an exponential response, corresponding to a first-order linear model. The vertical axis has been scaled for ease of comparison between both curves.

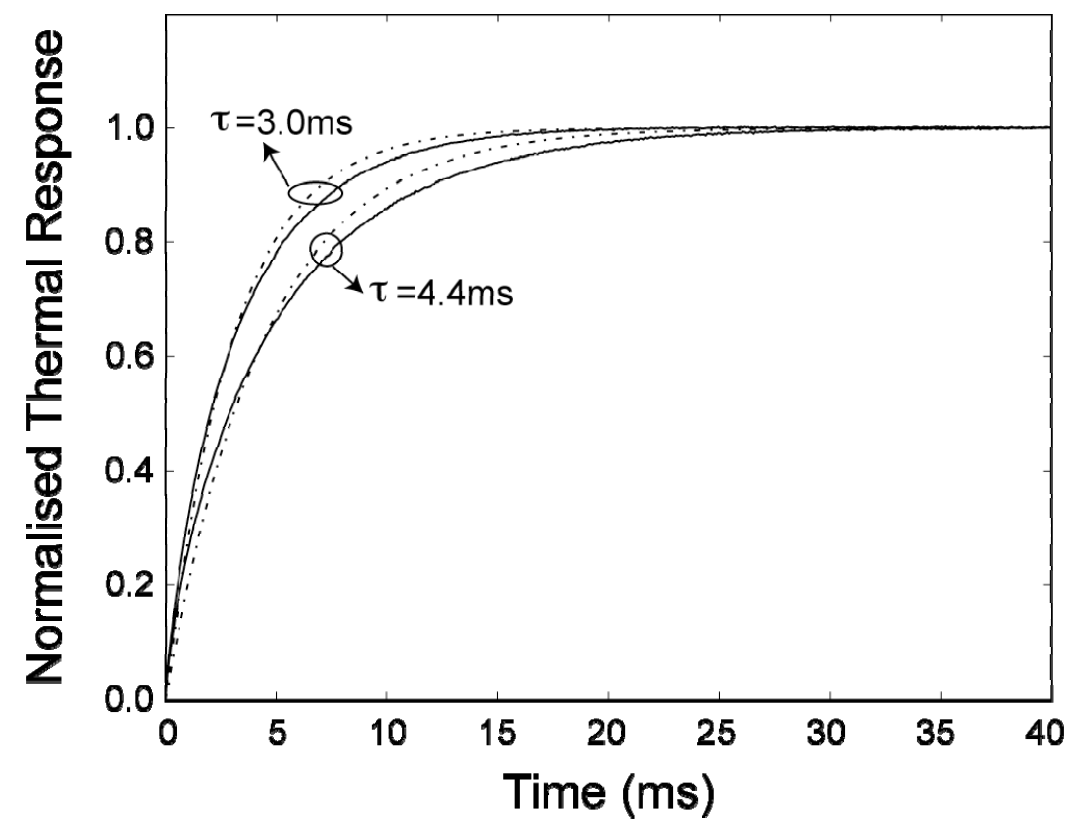

Fig. 10. Thermal step response of two membranes with widths $190 \mu \mathrm{m}$ and $240 \mu \mathrm{m}$ (the length is $2 \mathrm{~mm}$ ). The experimental results (continuous lines) are represented together with the best fit to an exponential response (broken lines), corresponding to a first order linear system. The smaller membrane has an estimated time constant of $3.0 \mathrm{~ms}$ and the larger membrane of $4.4 \mathrm{~ms}$ 
We see that the larger membrane ( $240 \mu \mathrm{m}$ vs $190 \mu \mathrm{m})$ exhibits a slower operational speed $(4.4 \mathrm{~ms}$ vs $3.0 \mathrm{~ms})$. The values of these time constants are relatively long, with the corresponding bandwidths at $36 \mathrm{~Hz}$ and $52 \mathrm{~Hz}$. This slow behavior is actually expected, due to the amorphous nature of the layers forming the membrane, which results in a very low thermal diffusivity. There is a deviation between the measurements and the first-order linear model. This deviation is not surprising, given that solving the heat equation for a membrane results in an additive series of exponential terms of different time constants even under a linearity assumption. Additionally the thermal diffusivity and conductivity are a function of temperature themselves, meaning that the linearity assumption does not hold for temperature variations larger than a few Kelvin degrees.

\section{CONCLUSIONS}

A novel concept of fast-scanning delay line making use of the thermo-optical effect has been developed and integrated with PECVD SiC rib waveguides to produce an ultra-compact TD-OCT engine. Although our current implementation still relies on external sources and detectors, the inclusion of these elements, together with driving and read-out electronics on the same die as the waveguides could enable extremely compact and low-cost TD-OCT solutions. This achievement should enable a wealth of new applications for OCT in the visible. The thermal response of the current design has been recorded and it has been found to exhibit bandwidths of a few tens of Hertz. This can be somewhat compensated for through preemphasizing the excitation signals and amplifying higher frequency components. Although this solution is helpful to some extent, for video-rate systems these devices are probably still too slow. There is significant room for improvements in speed through the introduction of high-diffusivity materials for thermal conduction and through new membrane dimensions. It is noteworthy that what is lost in terms of speed, is partially offset in terms of power efficiency. The low thermal diffusivity goes hand in hand with a low thermal conductivity, which limits the amount of power needed for a given temperature increase.

\section{ACKNOWLEDGMENT}

The authors are very thankful towards Bo Song and Marco Van der Krogt for handling the platinum deposition and towards Charles de Boer for advices on PECVD deposition.

\section{REFERENCES}

[1] G. Pandraud, P.M. Sarro and P.J. French, PDL free plasma enhanced chemical vapor deposition SiC optical waveguides and devices, Opt. Com., 269, 338-345 (2007)

[2] M. Brezinski, Optical Coherence Tomography: Principles and applications, Academic Press, 2006.

[3] V. Guedes, "Optical coherence tomography measurement of macular and nerve fiber layer thickness in normal and glaucomatous human eyes," Ophthalmology, 110, 1 (2003)

[4] I.-K. Jang, B.E. Bouma, D.H. Kang, S.-J. Park, S.-W. Park, K.-B. Seung, K.-B. Choi, M. Shishkov, K. Schlendorf, E. Pomerantsev, S.L. Houser, H.T. Aretz, and G.J. Tearney, "Visualization of coronary atherosclerotic plaques in patients using optical coherence tomography: comparison with intravascular ultrasound," J. Am. Coll. Cardiol., 39, 604-609 (2002)

[5] G. Pandraud, H.T.M. Pham, P.J. French and P.M. Sarro, "PECVD SiC optical waveguide loss and mode characteristics, “ Opt. Las. Technol., 39, 532-536,(2007)

[6] W. Drexler, U. Morgner, R. K. Ghanta, F. X. Kärtner, J. S. Schuman and J. G. Fujimoto' 'Ultrahighresolution ophthalmic optical coherence tomography," Nature Medicine 7, 502-507, (2001)

[7] L. Jiang, N. O. V. Plank, M. A. Blaw, R. Cheung, E. and van der Drift, (2004) "Dry etching of SiC in inductively coupled Cl2/Ar plasma" Journal of Physics D: Applied Physics, 37, 1809-1814 (2004)

[8] E. Margallo-Balbas, C.K. Yang, P.J. French, G. Pandraud, "Experimental characterization of roughness induced scattering los in $\mathrm{Si}$ and $\mathrm{SiC}$ wavegide sensors," Proc. IEEE Sensors, 1, 1557 -1561, (2009) 
[9] F. P. Payne and J. P. R. Lacey, "A theoretical analysis of scattering loss from planar optical waveguides,” Opt. Quant. Elec., 26, 977-986, (1994)

[10]W. K. Niblack, J. O. Schenk, B. Liu and M. E. Brezinski, "Dispersion in a grating-baesd optical delay line for optical coherence tomography," Appl. Opt., 42, 4115-4118, (2003)

[11]D. X. Xu, P. Cheben, D. Dalacu, A. Delage, S. Janz, B. Lamontagne, M. Picard and W. N. Ye, "Eliminating the birefringence in silicon-on-insolator ridge waveguides using the cladding stress," Opt. Lett. 29, 2384- 2386, (2004)

[12]P. Baeri, C. Spinella, R. Reitano, "Fast Melting of Amorphous Silicon Carbide Induced by Nanosecond Laser Pulse,” Int. J. of Thermophysics, 20, 1211-1221, (1999) 